\title{
Autopsies Findings and Evolution of the Therapeutic Strategies of the SARS-CoV-2
}

\section{Q Piacevoli ${ }^{1 *}$, L Marchioni ${ }^{2}$ and G Garotto ${ }^{2}$}

${ }^{1}$ San Filippo, Neri Hospital, Rome, Italy

${ }^{2}$ IRCCS Lazzaro Spallanzani Hospital, Rome, Italy

*Corresponding Author: Q Piacevoli, San Filippo, Neri Hospital, Rome, Italy.
Received: September 11, 2020

Published: October 30, 2020

(C) All rights are reserved by $\mathbf{Q}$ Piacevoli., et al.

\section{Abstract}

In light of the ongoing health emergency, we have launched a preliminary investigation into the alleged correlation between COVID-19 and all the other clinical manifestations.

SARS-CoV-2 has rapidly spread across the world, causing extensive morbidity and mortality, though the histopathologic basis of severe disease cases has yet to be studied in detail.

Autopsy has contributed significantly to our understanding of numerous disease processes, but for several reasons, autopsy reports following deaths related to SARS- CoV-2 have been limited.

Keywords: Autopsy; Inflammation; Thrombosis; Lungs; Heart

\section{Introduction}

The COVID-19 disease was first identified on December 31, 2019 by the health authorities of the city of Wuhan, capital of the Hubei province in China. The first SARS-CoV-2 outbreak in Italy was discovered on February 20, 2020 in Codogno (Lodi). Since then, our country has known 237,290 infected people and 34,371 deaths (data updated on May 16).

From the 22,792 published articles, it emerged that SARSCoV-2 not only causes damage to the respiratory system, but also to the cardiovascular system with arrhythmic, thromboembolic complications, heart failure, myocarditis, and disseminated intravascular coagulopathy, dermatitis.

Ling Mao., et al. on April 10, 2020 they published the first study concerning the alleged correlation between COVID-19 and neurological symptoms. In their case studies, 36.4\% of SARSCoV-2 patients developed neurological symptoms both in the central nervous system (dizziness, headache, altered state of consciousness, ataxia, epilepsy, cerebro-vascular disease) and in peripheral nervous system load (pain, dysaesthesia, dysgeusia and visual disturbances).

Our concern is that stroke in the patient with COVID-19 infection may be associated with a worse outcome (understood not only as an increase in mortality, but also as a greater neurological disability) compared to the patient with stroke only.

Coronavirus 2 from severe acute respiratory syndrome, abbreviated as SARS-CoV-2 (acronym from the English severe acute respiratory syndrome coronavirus 2), previously named new Coronavirus of 2019 (2019-nCoV, or even 2019 nCoV-ARD), is a viral strain of the SARS-related coronavirus/SARS-CoV-2 species, part of the genus Betacoronavirus (Coronaviridae family), subgenus Sarbecovirus, discovered around the end of 2019. It is the seventh coronavirus recognized as capable of infecting humans. The official name given by the World Health Organization to the syndrome caused by the virus is COVID-19 (abbreviation of the English Corona VIrus Disease-2019). 
Each SARS-CoV-2 virion has a diameter of approximately 50 200 nanometers [1-6].

Three-dimensional model of SARS-CoV-2 protein S (spike). Like other coronaviruses, SARS-CoV-2 has four structural proteins, known: as protein S (spike), E (envelope), M (membrane) and $\mathrm{N}$ (nucleocapsid); protein $\mathrm{N}$ contains the RNA genome while proteins $\mathrm{S}, \mathrm{E}$ and $\mathrm{M}$ together create the viral envelope. The spike protein, which has been analyzed at the atomic level by cryoelectronic microscopy, is the one that allows the virus to attach to the membrane of a host cell.

Understanding COVID-19: what does viral RNA load really mean? [7].

Nevertheless, based on the assumption that viral RNA load correlates with high levels of viral replication, there are important insights to be gained from this time-course analysis. Currently, our understanding of the relationship between viral RNA load kinetics and disease severity in patients with COVID-19 remains fragmented. That patients with COVID-19 with more severe disease requiring intensive care unit admission, had high viral RNA loads at 10 days and beyond, after symptom onset

\section{Materials and Methods}

The virus

SARS-CoV-2 [8] contains over 30,000 RNA bases. A proof-reading mechanism keeps this large genome from accumulating frequent mutations. The large spike protein $(\mathrm{S})$ forms a sort of crown on the surface of the viral particles. Its receptor-binding domain interacts with high affinity with angiotensin-converting enzyme 2 (ACE2) receptors on the surface of host cells. After the binding, two host cell proteases (Furin and TMPRLRS) cleave spike proteins and their exposed fusion peptides fuse the virus membrane with the membrane of the host cells. The virus RNA enters cells of the upper and lower respiratory tract, and it is translated into viral proteins. The cell dies releasing millions of new viruses that infect other cells and other individuals [9].

\section{The spreading}

There are various ways of SARS-CoV-2 spreading. The infection starts with the competition between the SARS-CoV-2 virions arrived in the respiratory mucosa that express high levels of ACE2 receptors and the barrier made by mucus secreted by goblet cells and moved by hair-like cilia and innate immunity reactions. There is evidence for the presence of the virus in cells other than respiratory epithelia, including gastrointestinal epithelial cells, endothelial cells and myeloid cells $[10,11]$.

\section{Immunity}

Cellular and humoral [12] innate immunity represents a first line of resistance which takes care of most encounters with infectious agents. Evidence from SARS-CoV-1 suggests that these viruses may block interferon-mediated antiviral immunity. CD8 cytotoxic $\mathrm{T}$ cells play a fundamental role in antiviral resistance. Evidence suggests that during COVID-19 T cells undergo functional exhaustion with lymphopenia, skewing towards a T17 phenotype, inappropriate for antiviral immunity and suppression $[13,14]$.

\section{Inflammation}

Inflammation plays a key role in the development of COVID-19 from a SARS-CoV-2 infection. Sensors of viral infection and cellular damage (e.g. Inflames some; TLR) trigger myeloid cell-dependent production of inflammatory cytokines (e.g. IL-1; IL6; chemokines). Macrophages and inflammatory cytokines amplify local and systemic inflammation and are major drivers of organ failure [15].

\section{Thrombosis}

Unsurprisingly for a disease characterised by an inflammatory state in response to a viral infection, venous and arterial thromboembolic complications are common in hospitalised patients. Micro thrombi are present in lungs, and alterations of the coagulation cascade can be measured at a systemic level. Endothelial dysfunction caused by both direct virus cytopathic effect and inflammatory reaction leads to a pro-thrombotic setting [16].

\section{First group of patients from Northern hospitals}

This report [17] want to describe the findings of the autopsies of the infected patients in order to improve the therapy and to have a better outcome. Pathological Anatomy Services have started performing autopsies on COVID19-positive patients, after some initial hesitation since their international scientific society has 
classified COVID-19 among the pathogens belonging to the hazard group 3 (like anthrax, prions, some mycobacteria... so to speak, Ebola belongs to $\mathrm{HG} 4$, which is the maximum level of danger). To date we have totaled about 150 autopsies, in almost all cases targeted to the lungs. We have waited for a special diving suit to be able to perform, even safely, the autoptic examination on the brain [18].

Ours is the largest case study in the world, since the Chinese have published only the results of 3 "minimally invasive" autopsies (+ another isolated case report) and another one from New Orleans ; the authors have published only other 3 cases. The only other Italian hospital that performs autopsies is the Sacco (with reports about 20 cases).

Here is what we learned of the first group of anatomicalpathological dissertation (presented by Gianatti and Sonsogni) and the most significant interventions of some of those present [19].

Already macroscopically the lungs appear "spotty", with hyperemic/hemorrhagic areas alternating with rosy areas. Histological, some areas are severely emphysematous, with enormously dilated blood vessels (up to 20 times the norm) often replacing microthrombi. In many cases, diffuse alveolar damage (DAD) is evident, with desquamation of pneumocytes, formation of hyaline membranes and a fibrotic exudate (as in ARDS). It appears as a high-flow syndrome, with hepatomegaly and dilated portal vessels, with diffuse thrombosis at all levels. Even the heart appears enlarged, always has a hydro pericardium and a marked left ventricular hypertrophy (but some of them are hypertensive patients).

In one case they observed a thrombus almost completely obstructing the superior vena cava and the right atrium. They have often noticed the ascent of the diaphragm, indicating that at a certain point the lungs no longer expand, associated with hepatomegaly. Waiting to have data on CNS samples, Sessa asked to biopsy the olfactory mucosa; COVID-19 classically gives anosmia and ageusia: it could reach the brain stem trans-synaptically starting from the peripheral nerve endings of the olfactory or lingual nerve (as well as from the innervation of the lungs). In this scenario, part of the respiratory failure could be caused by the direct damage of the virus on the nuclei of the brain stem (ambiguous nucleus, of the solitary tract...).

From the point of view of the cells of the immune system, many macrophages but very few lymphocytes intervene in the interstitium. Rambaldi points out that in the blood of patients with COVID-19 infection there is a very high number of endothelial cells (expression of the endothelial damage caused directly by the virus) and that these cells trigger a cytokine storm that mainly recruits macrophages. It is also for this reason that high-dose cortisone may work. An anti-complement drug (narsoplimab, the study is closed) was used on 6 patients which drastically reduced the number of circulating plasma endothelial cells (but clinical end-points were not specified).

In conclusion: The pathologists tell us to freely ask for the autopsies that we deem appropriate (especially sudden deaths, or deaths in relatively young and otherwise healthy patients), accompanied by as much information as possible (comorbidity, date of onset of symptoms, therapies carried out, 02 support system, transfer to TI). This is to understand how "past" there is in the disastrous lungs that have examined and to explain some anomalous findings (for example a case of amyloidosis or an abnormal thickening of the myocardium which according to Senni cannot be traced back to an acute myocarditis...).

In those days, Prof. L. Gattinoni [20] also reported that, perhaps, the classic ventilation of the ARDS at high pressures and the same prone-supination were in many cases useless when not harmful and of the utility of critically reviewing this attitude, marrying a ventilation as "gentle" as possible so as not to add iatrogenic damage to a disease for itself devastating... What appeared was not a classic ARDS.

At the same time, an increasing number of "sudden" deaths and more and more patients arriving exhausted from 15-20 days of serious home illness and with catastrophic intra-hospital course then accumulated in our unfortunate hospital.

From the anatomo-pathological data already described, it was possible to deduce some details that well explained why it was so difficult to ventilate lungs apparently affected by pneumonia in the 
ARDS phase (more than the classic ARDS): it was found, together with widespread alveolar damage with hyaline membranes, also important proliferation and exfoliation of type II pneumocytes, presence of inflammatory infiltrate mostly of the monocytemacrophage type and important early fibrosis phenomena induced by at least two lines: transformation in the fibrous sense of the pneumocytes and fibroblastic induction from the monocytes -macrophages; on the other side the presence of thrombotic (or thrombotic-hemorrhagic) micro-angiopathy phenomena.... The presence of lymphocytes was mostly scarce or in any case poorly represented. So "innate immunity" primary immunity and not/ little "secondary immunity", specific [21,22].

\section{Gathering some ideas I would like to try to summarize this} way: [23]

The virus enters our body with a certain affinity for the alveolar tissue but also for the vascular endothelial one, also widely represented in the lung but, obviously, it can (and we know for sure) take root in every organ and tissue. The initial viremic phase can run in a form that varies from total a symptomaticity to moderate to medium symptomaticity and has a variable duration: generally not very high fever $\left(<38.5^{\circ} \mathrm{C}\right)$, headache, widespread pain, rhinitis, conjunctivitis, little cough, little diarrhea or vomiting, anosmia.... At this point the inflammatory response occurs; if the NK and the first response is not sufficient to resolve the infection, a sort of second wave of symptoms much more aggressive than the previous ones can recur or, of course, overlap with the previous one: high fever, little responsive to paracetamol, persistent cough, ( any important gastrointestinal symptoms....). This phase is a so-called "hyperinflammation" characterized by an important elevation of the inflammation indexes with an impressive release of cytokines, high D-Dimer rates, etc... We could place at this level the invasion and macrophage activation that generates, on the one hand, the destruction of functional lung tissue, on the other extremely active "repair" -proliferation processes that are progressive fibrosis and on another side, the endothelial (-vascular) one, the progression of obliterating micro-angiopathy towards a form of proliferative thromboangioitis with promotion of thrombosis of the arterioles and then of the degree vessels themselves. The phenomenon is, at this point, (and can also be so from the beginning but more rarely) systemic so that extensive venous and arterial thrombotic phenomena can occur, concomitantly with changes also in the spontaneous coagulation and platelet profile, if not hindered in some way by direct action of drugs or measures. already widely remodeled, functionally inert, ten not vascularized due to the presence of multiple previous thromboembolic phenomena, therefore outcome.... In short, what we often radiologically (CT) refer to as diffuse interstitial pneumonia is already widely remodeled, functionally inert, often not vascularized due to the presence of multiple previous thromboembolic phenomena, therefore outcome [24].

We have observed, as the Hub of cardiovascular emergencies (STEMI- not STEMI), numerous coronary thromboses in the absence of significant atherosclerosis, more than expected, as well as numerous associated peripheral arterial thromboses, as well as DVT and TEP but, albeit to a lesser extent, hemorrhagic events only, perhaps, apparently unrelated to the disease... In fact, bleeding is easily macrophage direct activity of the mucous membranes after endothelial damage, at a relatively early stage (and sudden death was seen immediately after hospitalization for prolonged fever of a 43-year-old young woman due to gastro-duodenal bleeding) or it may be a late manifestation from a more complex thrombohemorrhagic vascular process, as we have seen in a case of collateral a. Pancreatic-duodenal bleeding aneurysm of collateral ligament stenosis arcuate of the celiac tripod, at the end of a long COVID pneumonia that mainly occurred at home (resolved fort together with success through embolization in urgency because the patient, a dear and very good Anesthesiologist-Resuscitator colleague one of the best in our hospital, had returned to the hospital for intractable abdominal pain).

Just one last consideration, but for a question that I personally feel extremely important: I think that much of what is exposed is potentially usable in the home patient, with few tools and above all with the refinement of the clinical question; this could make it possible to drastically reduce the arrival of patients to the hospital and above all the arrival at the end of a disease that has run substantially at home and is now often without real chances of recovery. 
Second group of patients with sudden death

\section{Clinical news [25]}

Bilateral interstitial pneumonia from Covid 19. ARDS. Kidney failure. Severe sepsis. Heart failure. The patients returned from Padua on March 6, 2020. Since then, fever has worsened on March 9, 2020. Admitted to the hospital s no COVID where they performs a chest CT scan that highlights multiple areas of bilateral interstitial infiltration. Hypertension in some. From blood chemistry tests anemia with hemoglobin 7.2, lymphopenia (from 14.5 to 2.2); LDH increased 353; transaminases and gamma GT increased (AST 161, ALT 200, LDH 439) alkaline phosphatase 127; fibrinogen 666; ferritin 463; platelets 146. Amylase 77 lipase 82.

\section{Macroscopic description [26]}

Asymmetry of the upper limbs with edema of the right upper limb continuous solution of the central region of the body as for tracheotomy Chest: Bilateral pleural effusion of about 1 It on the left and about $500 \mathrm{cc}$ on the right Pericardium: Opaque surface of the parietal sheet of the pericardium with growths referable to fibrin deposits as for fibrous pericarditis

\section{Heart [27]}

Heart of shape and volume preserved, concentric hypertrophy of both cardiac cavities is observed, with reduction of the lumen of the cavities. Thickening of the ventricle of the left ventricle wall of $2 \mathrm{~cm}$ and thickening of the wall of the right ventricle of $0.8 \mathrm{~cm}$; the myocardium appears pale, diminished in consistency and flabby; the atrial cavities appear dilated. Nothing to detect for the valve system. Ectasia of the ascending aorta. Lungs: Both lungs appear increased in volume and consistency; on the hilum the bronchi have hyperemic mucosa with catarrhal content, pulmonary arteries free of thrombi. Small bilateral hilar lymph nodes are observed. On the cutting surface, the lung parenchyma of both lungs has a compact appearance, an increased consistency, reddish complexion with areas of thickening of the interstitial bronchial vessel plot; when squeezed, frothy liquid flows out. In correspondence with the upper portion of the lower lobe of the left lung, a circumscribed area of compact hemorrhagic appearance is observed, well delimited with respect to the neighboring pulmonary parenchyma which takes on a relationship with the bronchial structures of the peripheral branches to be histologically ascertained [28].

\section{Abdomen}

Organs in place and normally positioned. Spleen: Preserved shape and volume, dimensions of $11 \times 4 \times 2 \mathrm{~cm}$. Thickened and opaque perisplenium. When cut, the pulp appears diffusely diffused. Liver: Form and volume preserved smooth Glissonian surface. On the cutting surface, I look like nutmeg due to the alternation of point yellowish and reddish areas. Gallbladder: Bile fluid. Adrenal: Histolytic degeneration of the medullary. Kidney: Easily encapsulated, external surface with reddish hollows. Clear corticomedullary limit. Esophagus: Hyperemia of the mucosa at the level of the esophagus-gastric junction Stomach: Digested food content; thinned mucosa. Pancreas: Adipose replacement Trachea: Hyperemic mucosa Brain: Nothing macroscopically relevant to the bones of the cranial vault; hemorrhagic petechiae of the dura mater, mainly in the right temporal area; hyperemia of leptomeninges. The cerebral hemispheres appear symmetrical, diminished in consistency. By performing sections along the coronary planes, we can appreciate modest atrophy of the cerebral cortex, a shiny white substance due to edema. Cerebral axis in axis, midbrain and cerebellum free from macroscopically detectable alterations. The vessels of the Willis circle show normal course and caliber.

\section{Macroscopic diagnosis [29]}

Cardiogenic shock from chronic pulmonary heart and acute pulmonary edema in subjects with bilateral interstitial foot pneumonia with circumscribed hemorrhagic neoformation of the left lung to be histologically ascertained. Cerebral edema. Chronic stasis liver. Acute passive congestion of the remaining organs.

\section{Third group of patients with sudden death}

We describe the first available large series of histopathological findings in patients died from COVID-19 in Rome Italy, with the aim to report the main microscopic pulmonary lesions in SARS-CoV-2 infection and severe respiratory failure.

\section{Methods}

This study is based on the histological analysis of post-mortem lung tissues from cases who died for COVID-19 between March and April. At the time of hospitalization, all the patients had throat swab sample positive for SARS-CoV-2 infection and had clinical 
and radiological features of interstitial pneumonia. D-dimer was available in all patients, with high value in all of them $(>10 \times$ the upper reference limit). histological examination, the features of Diffuse Alveolar Disease (DAD) 1) Macroscopic examination of the lungs revealed heavy, congested and oedematous organs, with spotty involvement. At histological examination, the features of Diffuse Alveolar Disease (DAD) were found, corresponding to those observable in the exudative and early/intermediate proliferative phases of the disease. Both phases often overlapped in the different areas of the lungs, with plurifocal pattern of distribution. The fibrotic phase was rarely observed. Moreover, five patients also had bacterial and fungal abscesses.

Capillary congestion, interstitial oedema, dilated alveolar ducts, hyaline membranes composed of serum proteins and condensed fibrin, loss of pneumocytes were the histological patterns of the exudative phase mostly observed in all the cases. Platelet-fibrin thrombi in small arterial vessels $(<1 \mathrm{~mm}$ in diameter) were found in 33 cases. Moreover, type II pneumocytes hyperplasia showing reactive atypia, myofibroblast proliferation, alveolar granulation tissue and obliterating fibrosis were present in half of the patients but were focal. Micro cystic honeycombing and mural fibrosis were occasionally present.

Inflammatory component [30] was represented by a few CD45 positive lymphocytes located in the interstitial space; a large number of CD68 positive macrophages were mainly localized in the alveolar lumens. Fibrin thrombi of small arterial vessels (diameter $<1 \mathrm{~mm}$ ) were observed in all patients [31].

\section{Results and Discussion}

After we performed a systematic literature search from all the most important data base and many autopsies we have started our protocol for the treatment for the Covid 19 patient from March 2020.

\section{Conclusion}

This report want to describe the findings of the autopsies of the infected patients in order to improve the therapy and to have a better outcome. It emerged that SARS-CoV-2 not only causes damage to the respiratory system, but also to the cardiovascular system

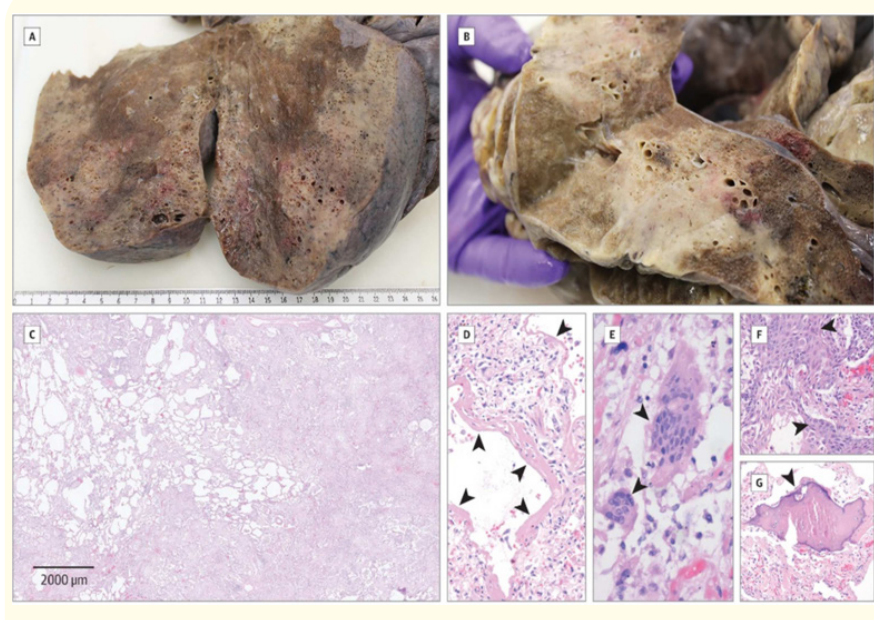

Figure a: Macroscopic (A and B) and histologic (C) images of organizing and end-stage diffuse alveolar damage (hematoxylineosin staining) with hyaline membranes (D, arrowheads, $\times 100$ ), multinucleated giant cells (E, arrowheads, $\times 400$ ), and squamous/ osseous metaplasia ( $\mathrm{F}$ and $\mathrm{G}$, arrowheads, $\times 200$ ) in a patient with a fatal course of coronavirus disease 2019.

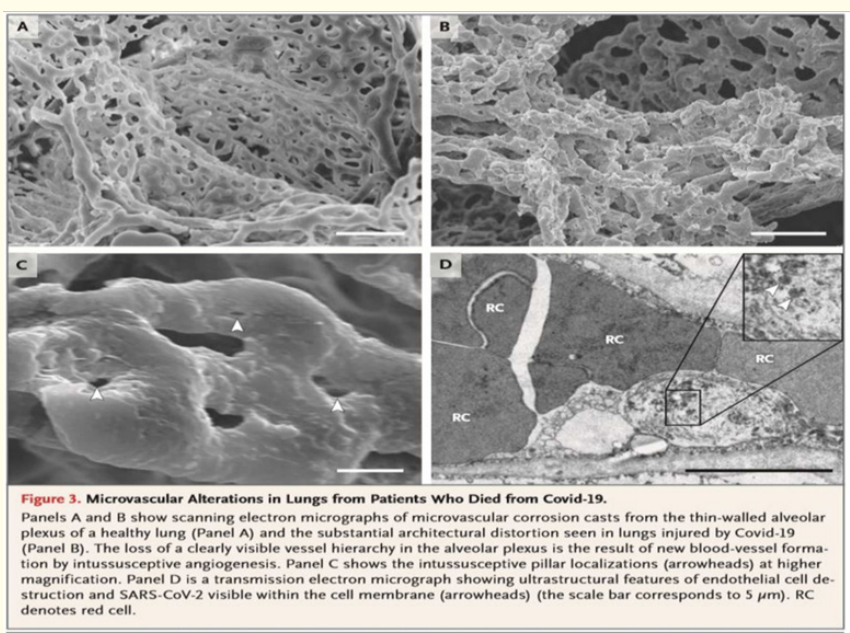

Figure b: Gross Findings of the Lungs and Heart. A) Lungs with bilateral pulmonary oedema and patches of dark hemorrhage, and B) A heart showing extreme right ventricular dilatation, with straightening of the interventricular septum. C) Cut sections of lung showing thrombi present within peripheral small vessels (white arrows). 

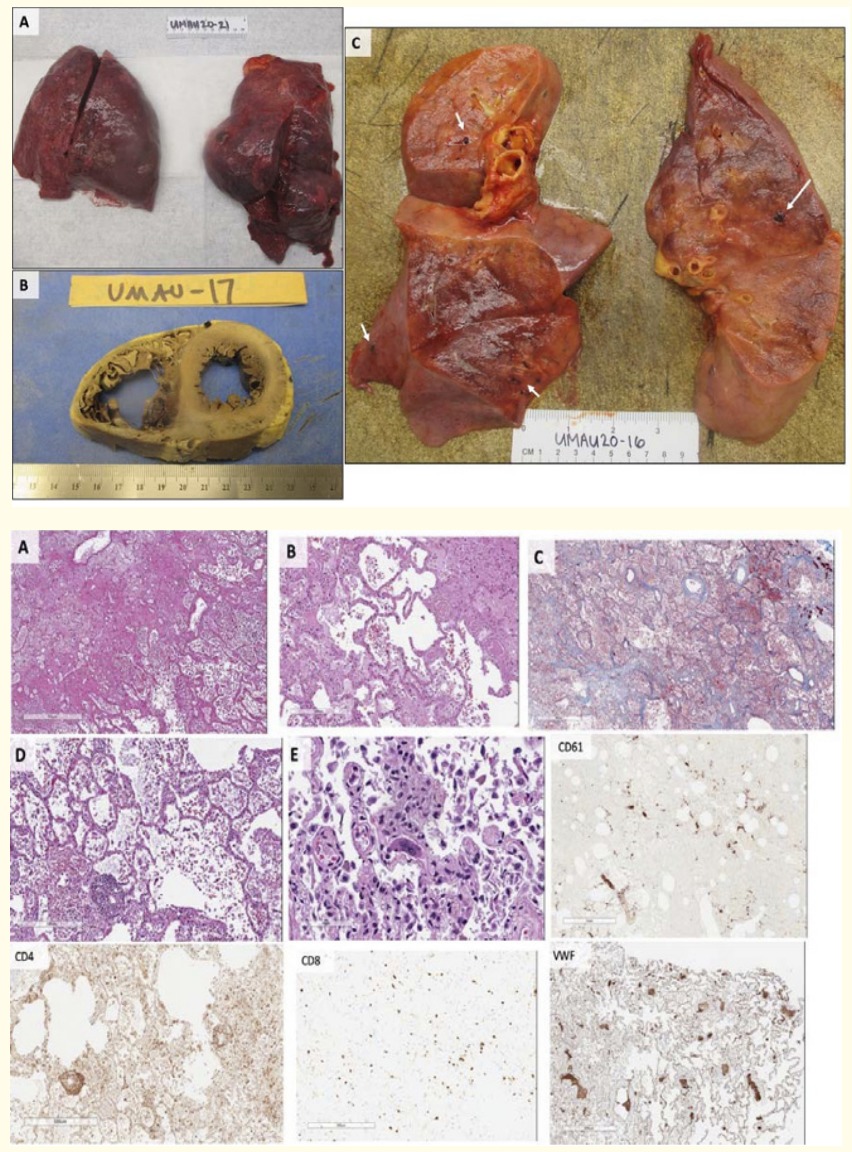

Figure c: A) Hyaline membranes and hemorrhage (H\&E), with B) Fibrin thrombi present within distended small vessels and capillaries, and C) Extensive extracellular fibrin deposition highlighted in blue D) Perivascular aggregations of lymphocytes, which were positive for CD4 immunostain, with only scattered CD8 positive cells present. E) Numerous megakaryocytes were present within the small vessels and alveolar capillaries, highlighted by CD61 and Von Willebrand Factor immunostains. First Autopsy series from New Orleans.

with arrhythmic, thromboembolic complications, heart failure, myocarditis, and disseminated intravascular coagulopathy, dermatitis.

\section{Conflict of Interest}

We don't have any financial interest or any conflict of interest.

\section{Bibliography}

1. $\mathrm{Wu} \mathrm{Z}$ and McGoogan JM. "Characteristics of and Important Lessons From the Coronavirus Disease 2019 (COVID-19) Outbreak in China". Summary of a Report of 72314 Cases From the Chinese Center for Disease Control and Prevention JAMA (2020).

2. Gaborit BJ., et al. "Plea for multitargeted interventions for severe COVID-19". Lancet Infection Disease 20 (2020): S14733099 (20)30312-1.

3. Lopinavit/ritonavir Scheda tecnica.

4. Kim Y., et al. "Reversal of the Progression of Fatal Coronavirus Infection in Cats by a Broad-Spectrum Coronavirus Protease". PLoS Pathogen 12.3 (2016): e1005531.

5. Jaegyun Lim., et al. "Case of the Index Patient Who Caused Tertiary Transmission of Coronavirus Disease 2019 in Korea: the Application of Lopinavir/Ritonavir for the Treatment of COVID-19 Pneumonia Monitored by Quantitative RT-PCR". Febbraio (2020).

6. Cao B., et al. "A Trial of Lopinavir-Ritonavir in Adults Hospitalized with Severe Covid-19". NEJM (2020).

7. Pak JE., et al. "Structural insights into immune recognition of the severe acute respiratory syndrome coronavirus S protein receptor binding domain". Journal of Molecular Biology 388.4 (2009): 815-823.

8. Ferretti L., et al. "Quantifying SARS-CoV-2 transmission suggests epidemic control with digital contact tracing". Science (2020).

9. Vabret N., et al. "Immunology of COVID-19: current state of the science". Immunity (2020). 
10. Lauer SA., et al. "The incubation period of coronavirus disease 2019 (COVID-19) from publicly reported confirmed cases: estimation and application". Annals of Internal Medicine 172.9 (2020): 577-582.

11. Young B E., et al. "Epidemiologic Features and Clinical Course of Patients Infected With SARS-CoV-2 in Singapore". JAMA 323.15 (2020): 1488-1494.

12. Hwang WC., et al. "Structural basis of neutralization by a human anti-severe acute respiratory syndrome spike protein antibody, 80R". Journal of Biological Chemistry 281.45 (2006): 34610-34616.

13. Loria GD., et al. "Platelets support a protective immune response to LCMV by preventing splenic necrosis". Blood 121.6 (2013): 940.

14. Li F., et al. "Structure of SARS corona- virus spike receptorbinding domain complexed with receptor". Science 309.5742 (2005): 1864-1868.

15. Chan JF., et al. "Genomic characterization of the 2019 novel human-pathogenic coronavirus isolated from a patient with atypical pneumonia after visiting Wuhan". Emergency Microbes Infection 9.1 (2020): 221-236.

16. Varga Z., et al. "Endothelial cell infection and endotheliitis in COVID- 19". Lancet 395.10234 (2020): 1417-1418.

17. Lodigiani C., et al. "Venous and arterial thromboembolic complica- tions in COVID-19 patients admitted to an academic hospital in Milan, Italy". Thrombosis Research 191 (2020): 9-14.

18. Grasselli G., et al. "Critical care utilization for the COVID-19 outbreak in Lombardy, Italy: early experience and forecast during an emergency response". JAMA 323.16 (2020): 15451546.

19. Joly BS., et al. "Understanding pathophysiology of hemostasis disorders in critically ill patients with COVID-19". Intensive Care Medicine (2020).
20. L Carsana., et al. "Pulmonary post- mortem findings in a large series of COVID -19 cases from Northern Italy". medRxiv (2020).

21. Gattinoni L., et al. "Covid-19 does not lead to a "typical" acute respiratory distress syndrome". American Journal of Respiratory and Critical Care Medicine 201.10 (2020): 12991300.

22. Marini JJ and Gattinoni L. "Management of COVID-19 respiratory distress". JAMA (2020).

23. Schultz MJ. "High versus low PEEP in non-recruitable collapsed lung tissue: possible implications for patients with COVID-19". Lancet Respiratory Medicine 8 (2020): e44.

24. Tobin MJ. "Principles and practice of mechanical ventilation". 3rd ed. New (2020).

25. Prabakaran P., et al. "Structure of severe acute respiratory syndrome coronavirus receptor- binding domain complexed with neutralizing anti-body". Journal of Biological Chemistry 281.23 (2006): 15829-15836.

26. Franks T., et al. "Lung pathology of severe acute respiratory syndrome (SARS): a study of 8 autopsy cases from Singapore". Human Pathology 34 (2003): 643-748.

27. Alsaad KO., et al. "Histopathology of Middle East respiratory syndrome coronavirus (MERS-CoV) infectionClinicopathological and ultrastructural study". Histopathology 72 (2018): 516-524.

28. Sharon E., et al. "Pulmonary and Cardiac Pathology in Covid-19: The First Autopsy Series from New Orleans”. medRxiv (2020).

29. Chen L., et al. "The ACE2 expression in human heart indicates new potential mechanism of heart injury among patients infected with SARS-COV-2". Cardiovascular Research (2020).

30. Campbell RA., et al. "Human megakaryocytes possess intrinsic antiviral immunity through regulated induction of IFITM3". Blood 133.19 (2019): 2013-2026. 
31. Yang M., et al. "Thrombocytopenia in patients with severe acute respiratory syndrome (review)". Hematology 10.2 (2005): 101-105.

\section{Assets from publication with us}

- Prompt Acknowledgement after receiving the article

- Thorough Double blinded peer review

- Rapid Publication

- Issue of Publication Certificate

- High visibility of your Published work

Website: www.actascientific.com/

Submit Article: www.actascientific.com/submission.php

Email us: editor@actascientific.com

Contact us: +919182824667 